\title{
Anti-inflammatory effect of probiotic Lactobacillus paracasei MSMC39-1 on alcohol-induced hepatitis in rats
}

\author{
Boonyarut Ladda ${ }^{1}$, Piyatida Tangteerawatana' ${ }^{1}$ Poolpol Padungchaichot ${ }^{2}$, Wisuit Pradidarcheep ${ }^{2}$, Anongnard Kasorn ${ }^{3}$, \\ Malai Taweechotipatr ${ }^{*}$ \\ ${ }^{1}$ Department of Microbiology, Faculty of Medicine, Srinakharinwirot University, Bangkok, Thailand. \\ ${ }^{2}$ Department of Anatomy, Faculty of Medicine, Srinakharinwirot University, Bangkok, Thailand. \\ ${ }^{3}$ Department of Basic Medical Science, Faculty of Medicine Vajira Hospital, Navamindradhiraj University, Bangkok, Thailand.
}

\begin{tabular}{|c|c|}
\hline ARTICLE INFO & ABSTRACT \\
\hline $\begin{array}{l}\text { Received on: } 26 / 10 / 2020 \\
\text { Accepted on: } 14 / 02 / 2021 \\
\text { Available online: } 05 / 04 / 2021\end{array}$ & $\begin{array}{l}\text { Probiotics are good microbiota which are able to promote the health of host immunomodulation. In this study, we } \\
\text { prepared four antigens of Lactobacillus paracasei MSMC39-1 for the detection of tumor necrosis factor- } \alpha \text { (TNF- } \\
\alpha \text { ) secretion in hepatocellular carcinoma cell line (HepG-2). We found that viable cells and heat-killed cells of } L \text {. } \\
\text { paracasei MSMC39-1 exhibited strong TNF- } \alpha \text { secretion inhibitors. Viable cells of L. paracasei MSMC39-1 treatment }\end{array}$ \\
\hline $\begin{array}{l}\text { Key words: } \\
\text { Probiotics, hepatocellular } \\
\text { carcinoma cell line, alcoholic } \\
\text { hepatitis, tumor necrosis } \\
\text { factor, Lactobacillus } \\
\text { paracasei. }\end{array}$ & $\begin{array}{l}\text { significantly reduced serum level of aspartate aminotransferase in hepatitis rats. TNF- } \alpha \text { secretion in liver tissues of } \\
\text { hepatitis rats in probiotic } L \text {. paracasei MSMC39-1 treatment was significantly suppressed. Liver histopathology } \\
\text { examination of alcohol-induced hepatitis indicated fat accumulation in the hepatocytes. Interestingly, liver damage in } \\
\text { hepatitis rats was improved by probiotic } L \text {. paracasei MSMC39-1. In addition, L. paracasei MSMC39-1 alleviated } \\
\text { colon inflammation in alcohol-induced hepatitis rats. Administration of L. paracasei MSMC39-1 modulated gut } \\
\text { microbiota by increasing the number of genera Lactobacillus and Bifidobacterium in alcohol-induced hepatitis rats. } \\
\text { We conclude that } L \text {. paracasei MSMC39-1 has potent anti-inflammatory effects on HepG-2 cell line and improves } \\
\text { hepatitis, colon inflammation, and modulates gut microbiota. Thus } L \text {. paracasei MSMC39-1 is able to protect against } \\
\text { alcoholic hepatitis. It can also be used as an alternative treatment in other inflammation diseases and applied in } \\
\text { nutraceutical and dietary supplement products. }\end{array}$ \\
\hline
\end{tabular}

\section{INTRODUCTION}

Probiotics, beneficial microbiota exhibit several health benefits in human diseases including alcoholic liver disease, obesity, allergy, and inflammatory bowel disease (IBD) (Fang et al., 2019; Huang et al., 2019; Rabah, 2020; Wang et al., 2017). Several studies have utilized probiotics to compensate and reduce side effects from the use of antibiotics and as health supplements. In addition, probiotics are used in animal food products in many countries. Food and Agriculture Organization of the United Nations and World Health Organization (FAO and WHO) defines

${ }^{*}$ Corresponding Author

Malai Taweechotipatr, Department of Microbiology,

Faculty of Medicine, Srinakharinwirot University. Bangkok, Thailand.

E-mail:malai@g.swu.ac.th probiotics as "live microorganisms which when administered in adequate amounts confer a health benefit on the host" (WHO/FAO, 2002). Selection criteria of probiotics include toleration to acid and bile, adhesion in gut epithelial cell, production of antimicrobial substances, and modulation of immunity. Safety of probiotics follows the criteria of Generally Recognize as Safe by the United States Food and Drug Administration (USFDA) (Shewale et al., 2014). According to their properties, probiotics are considered as a member of good microbiota with health benefits.

Genera of Lactobacillus, Bifidobacterium, Enterococcus, Streptococcus, Bacillus, Lactococcus, and Saccharomyces are used as probiotics. Probiotics have been reported to enhance tight junction, decrease cholesterol level with bile salt hydrolase production, produce gamma-aminobutyric acid (GABA), and modulate proinflammatory cytokines (Bermudez-Brito et al., 2012; Das and Goyal, 2015). Tumor necrosis factor (TNF), a major proinflammatory cytokine, plays a key role in the inflammation 
process. TNF are divided into two forms: tumor necrosis factor- $\alpha$ $(\mathrm{TNF}-\alpha)$ and TNF-beta (TNF- $\beta$ ). TNF- $\alpha$ is mainly produced by macrophage. Binding between TNF and TNF receptor 1 leads to transcription of genes encoding proinflammatory cytokines, chemokines, cell survival factors, and growth factors via Ikappa B $(\mathrm{I} \kappa \mathrm{B})$ kinase $(\mathrm{IKK}) /$ nuclear factor kappa-B $(\mathrm{NF}-\kappa \mathrm{B})$ and mitogenactivated protein kinase /AP-1 pathways (Cheung and Ware, 2013). The level of TNF is alleviated in patients with inflammatory conditions including IBD, rheumatoid arthritis, alcoholic liver disease, atopic dermatitis, and psoriasis (Chu, 2013). Alcoholic hepatitis is a disease caused by chronic alcohol consumption. It causes accumulation of fat and toxin in liver, disruption of intestinal barrier, and stimulation of proinflammatory cytokines leading to liver inflammation and dysbiosis in gastrointestinal (GI) tract. Lipopolysaccharide (LPS) which is released from bad microbiota due to chronic ethanol consumption binds to toll-like receptor 4 (TLR-4) and activates Kupffer cells in liver tissue. Kupffer cells then secrete proinflammatory cytokines such as TNF- $\alpha$, interleukin (IL)- $1 \beta$, and IL- 6 and generate reactive oxygen species via NF- $\mathrm{kB} / \mathrm{JNK} / \mathrm{AP}-1$ pathway (Fung and Pyrsopoulos, 2017). Drug is commonly used as a treatment for liver diseases to relieve symptoms of patients, and liver transplantation is the last treatment manner (Leggio and Lee, 2017).

Probiotics are used as an alternative treatment for liver disease. Several pieces of evidence both in vitro and in vivo reported the usage of probiotics in this disease. Probiotic lactic acid bacteria reduced TNF- $\alpha$ and IL- 6 secretion in LPS stimulated human hepatocellular carcinoma cells (HepG2) (Chiu et al., 2013). Lactobacillus rhamnosus GG and its component improved TLRs and suppressed proinflammatory cytokine of LPS treated porcine intestinal epithelial cell via NF-kB signaling pathway (Gao et al., 2017). In addition, Bifidobacterium bifidum, Lactobacillus casei, and Lactobacillus plantarum fermented soymilk alleviated liver injury and reduced TNF in liver tissue of rat fed high-fat diet (Zhang et al., 2017). Probiotic Bifidobacterium animalis reduced liver injury which reduced mRNA level of TNF- $\alpha$ in mice with alcoholic liver disease (Zhang et al., 2019). Hong et al. (2015) reported that L. rhamnosus and Lactobacillus acidophilus decreased levels of TNF- $\alpha$, IL-1 $\beta$, and TLR-4 expression and increased anti-inflammatory cytokine (IL-10) in an alcoholic hepatitis mouse model (Hong et al., 2015). Pretreatment of probiotic $L$. casei reduced TNF- $\alpha$, IL- $1 \beta$, endotoxin production, hepatic inflammation, and increasing of genus Lactobacillus and Bifidobacterium in feces of LPS and D-galactosamine induced acute liver failure rats (Wang et al., 2016). The previous study on mice model showed that L. rhamnosus GG supplementation reduced hepatic inflammation and TNF- $\alpha$ production via inhibition of TLR-4 and TLR-5 activation in $5 \%$ alcoholinduced liver injury for 8 weeks (Wang et al., 2013). Likewise, the study on L. rhamnosus GG (LGG) supplementation reduced liver inflammation by protected gut microbiota alteration for the treatment of alcoholic liver disease (Bull-Otterson et al., 2013). A recent study on alcoholic liver injury rats showed that L. plantarum C88 reduced alanine aminotransferase (ALT) and aspartate aminotransferase (AST) enzymes in the liver and decreased TNF- $\alpha$, IL-6, and interferon-gamma (IFN- $\gamma$ ) in serum. Moreover, C88 inhibited NF- $\kappa \mathrm{B}$ inflammation signaling pathway (Zhao et al., 2017). Similarly, L. plantarum and Lactobacillus fermentum treatment reduced ALT and AST levels in serum and decreased TNF- $\alpha$ and IL-1 $\beta$ gene expression in hepatic tissue of carbon tetrachloride-induced acute liver injury mice model (Chen et al., 2018). A recent study on acute liver injury rat model induced by D-galactosamine injection shows that pretreatment of Lactobacillus helveticus decreased ALT and AST level in the serum and was downregulated TNF- $\alpha$ gene expression in the hepatic tissue (Wang et al., 2019). Our previous data suggested that L. paracasei MSMC39-1 and L. confusus MSMC57- 1 strongly inhibited TNF- $\alpha$ secretion in human monocytic cell line (THP-1) (Ladda et al., 2015). Therefore, the aim of this study is to investigate the roles of probiotic L. paracasei MSMC39-1 in the suppression of TNF- $\alpha$ secretion in hepatocellular carcinoma cells and alleviated liver inflammation in alcohol-induced hepatitis rats. We hope to search for probiotic Lactobacillus which is able to be a potential candidate for ameliorating of liver inflammation.

\section{MATERIALS AND METHODS}

\section{Probiotic strains}

The probiotic L. paracasei MSMC 39-1 and L. confusus MSMC57-1 were used in this study. These strains were isolated from newborn feces (SWUEC 37/2551) which inhibited TNF- $\alpha$ production in the THP-1 monocytic cell line (Ladda et al., 2015).

\section{Cultivation of probiotics}

Lactobacillus paracasei MSMC39-1 and L. confusus MSMC57-1 were cultured in deMan-Rogosa-Sharpe (MRS) broth (Himedia, India) and incubated at $37^{\circ} \mathrm{C}$ for $48 \mathrm{~h}$ under anaerobic condition (Whitley Jar Gassing System, UK) in an anaerobic jar (Mitsubishi, Japan). Subsequently, the probiotic culture was streaked on MRS agar supplemented with $0.3 \%$ calcium carbonate (Merck, USA) and incubated at $37^{\circ} \mathrm{C}$ for $48 \mathrm{~h}$ under anaerobic condition. The overnight culture of L. paracasei MSMC39-1 and $L$. confusus MSMC57-1 was picked up as a single colony and added to MRS broth and incubated at $37^{\circ} \mathrm{C}$ for $48 \mathrm{~h}$ under anaerobic condition for the next experiments.

\section{Preparation of probiotic antigens}

\section{Probiotic culture supernatant and viable cells}

Lactobacillus paracasei MSMC39-1 and L. confusus MSMC57-1 were cultured on MRS agar and incubated at $37^{\circ} \mathrm{C}$ under anaerobic condition for $48 \mathrm{~h}$. The single pure colony was picked up and inoculated in MRS broth and incubated at $37^{\circ} \mathrm{C}$ under anaerobic condition for 48 hours. The two strains were adjusted to a final concentration of $10^{9}$ colony forming units (CFU)/ml (Ladda et al., 2015). The supernatant was separated by centrifugation and filtered with $0.22 \mu \mathrm{m}$ and concentrated by a vacuum concentrator (Christ, Germany) at $40^{\circ} \mathrm{C}$ for $4-6$ hours. The concentrated supernatant was resuspended in serum-free-medium Dulbecco's modified eagle medium (DMEM) and kept at $-20^{\circ} \mathrm{C}$ until used. For viable cell preparation, the final concentration of viable cell pellet was washed twice with phosphate buffer saline (PBS). The pellets were resuspended with serum-free DMEM and used immediately for TNF inhibition test in cell lines.

\section{Heat-killed cells}

Lactobacillus paracasei MSMC39-1 and L. confusus MSMC57-1 were cultured on MRS agar, anaerobically incubated 
at $37^{\circ} \mathrm{C}$ for 48 hours. The single colony was cultured in MRS broth and incubated at $37^{\circ} \mathrm{C}$ in anaerobic condition for 48 hours. The probiotic strains were adjusted to the final concentration of $10^{9} \mathrm{CFU} / \mathrm{ml}$ (Ladda et al., 2015), centrifuged, and washed twice with PBS. The pellets were resuspended in PBS, heated at $85^{\circ} \mathrm{C}$ for 1 hour, and kept at $-20^{\circ} \mathrm{C}$ until used. The cell viability was rechecked by streaking on MRS agar and anaerobically incubated at $37^{\circ} \mathrm{C}$ for 48 hours.

\section{Sonicated cells}

Lactobacillus paracasei MSMC39-1 and L. confusus MSMC57-1 were cultured on MRS agar incubated at $37^{\circ} \mathrm{C}$ anaerobic for 48 hours. The single colony was picked up and cultured in MRS broth and incubated at $37^{\circ} \mathrm{C}$ in anaerobic condition for 48 hours. The probiotic strains were adjusted to the final concentration of $10^{9} \mathrm{CFU} / \mathrm{ml}$ (Ladda et al., 2015), centrifuged, and washed twice with PBS. The pellets were resuspended in serumfree medium DMEM. Resuspended cells were sonicated at the set speed of $85 \%-88 \%$ for 7.10 minutes by ultrasonic homogenizers (Sonopuls, Bandelin, Germany) and kept at $-20^{\circ} \mathrm{C}$ until used.

\section{Cell culture}

HepG-2 cells (HB 8065) and hepatocellular carcinoma cell line were purchased from American Type Culture Collection (ATCC). HepG-2 cells were cultured in DMEM containing 10\% fetal bovine serum and $1 \%$ penicillin (100 units $/ \mathrm{ml}$ ) streptomycin $(100 \mathrm{mg} / \mathrm{ml})$ and incubated in $37^{\circ} \mathrm{C}$ incubator with $98 \%$ humidified and $5 \% \mathrm{CO}_{2}$ (Chiu et al., 2013).

\section{Determination of TNF- $\alpha$ secretion in HepG-2 cells}

Hepatocellular carcinoma cells were seeded at $1 \times 10^{5}$ cells/well on a 24-well plate for 3 days (Chiu et al., 2013). Cells were treated with $10 \% \mathrm{v} / \mathrm{v}$, probiotic culture supernatant, $20 \% \mathrm{v} / \mathrm{v}$ heat-killed cells, and sonicated cells or viable cells of $L$. paracase $i$ MSMC39-1 and L. confusus MSMC57-1 and stimulated with a final concentration of $5 \mu \mathrm{g} / \mathrm{ml}$ purified LPS from Escherichia coli serotype O127:B8 (Sigma, USA) for 48 hours. Collection of supernatant was centrifuged at $1,000 \mathrm{~g}, 4^{\circ} \mathrm{C}, 5$ minutes. Levels of TNF- $\alpha$ in the supernatants were detected by human TNF- $\alpha$ sandwich enzyme-linked immunosorbent assay (ELISA) kit according to the manufacturer's instructions (R\&D Systems, Minneapolis, MN).

\section{Experimental animals}

Male Wistar rats (Rattus norvegicus), aged 8 weeks and weighing 200-250 g, were purchased from Namura Siam International (Thailand). Housing of the animals was controlled at a temperature of $22 \pm 2{ }^{\circ} \mathrm{C}$, humidity: $55 \% \pm 5 \%$, and $12: 12$ hours light/dark cycle at Medical Center Animal Care Laboratory, Srinakharinwirot University, Thailand. The rats were adapted to the environment for 1 week before the experiment. The animal experiment obtained informed license no. 9/2561 from the Animal Ethics Committee of the Faculty of Medicine, Srinakharinwirot University.

\section{Induction of hepatitis}

Alcoholic hepatitis was induced in each rat by oral gavage with $30 \%$ ethanol dissolved in reverse osmosis water at a dose of $5 \mathrm{mg} / \mathrm{kg}$ of body weight for 8 weeks (Forsyth et al., 2009; Zhao et al., 2017).

\section{Experimental designs}

Lactobacillus paracasei MSMC39-1 was the strongest TNF- $\alpha$ inhibition. Thus, L. paracasei MSMC39-1 was selected for the animal experiment. The rats were divided into four groups of six animals each. Standard diet and distilled water were given to rats in the normal control group (group 1). Viable cells of probiotic L. paracasei MSMC39-1 with standard diet were administrated to rats in the probiotic control group (group 2). Hepatitis rats (group 3) received a standard diet with $30 \%$ alcohol. Probiotictreated rats (group 4) received a standard diet with $30 \%$ alcohol and viable cells of $L$. paracasei MSMC39-1. Viable cells of $L$. paracasei MSMC39-1 were administered before induction of hepatitis for 2 weeks and treated continuously until 8 weeks; the standard diet was ad libitum. Viable cells of L. paracasei MSMC $39-1$ were given to rats in groups 2 and 4 at $1 \times 10^{9} \mathrm{CFU} / \mathrm{ml} / \mathrm{rat} /$ day. At the end of the experiment, body and liver weight were measured in each rat. Then rats were anesthetized with isoflurane, and blood was collected from cardiac puncture.

\section{Detection of liver function enzymes}

Rats were anesthetized with isoflurane, and blood was collected from cardiac puncture at the end of the experiment (Wang et al., 2016). Serum was separated and centrifuged at 1,500 g, $4^{\circ} \mathrm{C}$ for 10 minutes. AST and ALT levels were detected in the serum (Zhao et al., 2017).

\section{Detection of TNF- $\alpha$ level in the liver}

After rats were euthanasia, liver tissue was removed, weighed, and homogenized by ultrasonic homogenizers (Sonopuls, Bandelin, Germany) at 35\% power and centrifuged at $800 \mathrm{~g}, 5$ minutes, $4^{\circ} \mathrm{C}$. Levels of TNF- $\alpha$ in the supernatant of liver homogenate were detected by sandwich ELISA kit according to the manufacturer's instructions (R\&D Systems, Minneapolis, MN) (Ladda et al., 2015).

\section{Histology evaluation of hepatitis rat}

After treatment, rats were anesthetized with isoflurane and sacrificed. Liver and colon were removed, weighed, and excised from the tissue (Wang et al., 2016). The pieces of tissue were fixed in $4 \%$ paraformaldehyde at $4{ }^{\circ} \mathrm{C}$ and dehydrated in a graded alcohol series $(10 \%, 20 \%, 50 \%, 95 \%$, and $100 \%)$. The liver and colon specimens were embedded in paraffin and sectioned at 5-7 $\mu \mathrm{m}$. The section slides were deparaffinized, cleared in xylene, and rehydrated in a graded series of ethanol $(100 \%, 95 \%$, and $70 \%)$ before staining with hematoxylin and eosin (H\&E). Histology of each tissue section was evaluated under a light microscope (Olympus UC50, Japan).

\section{Detection of fecal microbiota by standard plate count}

Stool was collected at the end of the experiment for detection of gut microbiota alteration. One gram of stool was diluted in normal saline solution and spread in MRS agar, Bifidobacterium agar, and Eosin Methylene Blue Agar (EMB agar). The plates were incubated at the optimal condition of each 
bacterial growth. Colonies were counted and calculated as the percentage of fecal microbiota alteration.

\section{Detection of gut microbiota by next-generation sequencing}

Stool was collected at the end of the experiment for detection of gut microbiota alteration by next-generation sequencing. QIAamp Fast DNA Stool Mini Kit was used for stool DNA extraction according to the manufacturer's instructions (Qiagen, Germany). DNA concentration was measured by Nanodrop 2000 spectrophotometer (Thermo Scientific, Waltham, MA). The primers used in this study were as followed: forward primer: 5'TCGTCGGCAGCGTCAGATGTGTATAAGA

GACAGCCTACGGGNGGCWGCAG3' and reverse primer: 5'GTCTCGTGGGCTCGGAGATG

TGTATAAGAGACAGGACTACHVGGGTATCTAAT CC3'. Two variable regions of $16 \mathrm{~S}$ rDNA (V3 and V4) of $16 \mathrm{~S}$ metagenome were sequenced by Illumina Miseq sequencer (Illumina, San Diego, CA) (Pérez-Burillo et al., 2020).

\section{Statistical analysis}

Statistical significances or differences were evaluated by GraphPad Prism version 8.00. For the in vitro study, Student's $t$-test with one-tailed distribution was used. A $\rho$-value $\leq 0.05$ was considered to be statistically significant. For the animal study, oneway analysis of variance (ANOVA) comparing each group with the control group was used and expressed as mean \pm SD.

\section{RESULTS AND DISCUSSION}

\section{The effect of $L$. paracasei MSMC39-1 on the TNF- $\alpha$ secretion in HepG-2 cells}

Probiotic lactic acid bacteria mostly genus Lactobacillus and Bifidobacterium have been used to promote human health. Probiotic Lactobacillus isolated from human, fermented foods, and dairy products exhibited antimicrobial, anticancer, antioxidant, and cholesterol-lowering properties as well as immunomodulation (Choi and Chang, 2015; Kim et al., 2019; Miao et al., 2020; Pan et al., 2020). Alleviation of TNF- $\alpha$ can be found in inflammatory diseases such as IBD, rheumatoid arthritis, alcoholic liver disease, atopic dermatitis, and psoriasis. TNF- $\alpha$ is also detected in alcohol liver disease and plays a key role in liver inflammation (Niederreiter and Tilg, 2018). Inhibition of TNF- $\alpha$ production by probiotics has been reported. L. rhamnosus, 4B15, and Lactobacillus gasseri 4M13 isolated from human feces with antioxidant and cholesterol reduction properties suppressed the production of proinflammatory cytokine including TNF- $\alpha$, IL-6, and IL-1 $\beta$ in RAW 264.7 macrophage cell line (Oh et al., 2018). In addition, surface layer protein of L. acidophilus decreased inflammatory cytokine secretions including TNF- $\alpha$, IL-10, IL-1 $\beta$, interferon-alpha (IFN- $\alpha$ ), and interferon-beta (IFN- $\beta$ ) in LPSactivated RAW 264.7 cells (Cai et al., 2018).

Four antigens including probiotic culture supernatant, viable cells, heat-killed cells, and sonicated cells were prepared from L. paracasei MSMC39-1 and L. confusus MSMC57-1. The effects of these four antigens on TNF- $\alpha$ were investigated in LPS-induced TNF- $\alpha$ secretion in HepG-2 cells. Although probiotic culture supernatant of MSMC57-1 had no effect on TNF- $\alpha$ secretion, a slight inhibition of TNF- $\alpha$ secretion can be observed in HepG-2 cells treated with probiotic culture supernatant of L. paracasei MSMC39-1 (Fig. 1A). Viable cells of L. paracasei MSMC39-1 and L. confusus MSMC57-1 decreased TNF- $\alpha$ secretion in LPS-activated HepG-2 cells with 53\% and $23 \%$ reduction, respectively (Fig. 1B). More than $50 \%$ of TNF- $\alpha$ secretion was suppressed by heat-killed cell of $L$. paracase $i$ MSMC39-1 when compared to MRS controls $\left({ }^{*} p<0.05\right)$ (Fig. $1 \mathrm{C})$. TNF- $\alpha$ secretion was comparable in HepG-2 cells treated with heat-killed cells of $L$. confusus MSMC57-1 when compared with the MRS control (Fig. 1C). Sonicated cells of L. confusus MSMC57-1 significantly suppressed TNF- $\alpha$ secretion in HepG-2 cells $(* p<0.05)$ (Fig. 1D). Slight inhibition of TNF- $\alpha$ secretion was observed in HepG-2 cells treated with L. paracasei MSMC391 (Fig. 1D). In this study, four antigens generated from probiotic lactic acid bacteria, L. paracasei MSMC39-1 and L. confusus MSMC57-1, were used to investigate the TNF- $\alpha$ secretion in HepG-2 cells. The supernatant of L. paracasei MSMC39-1 slightly inhibited TNF- $\alpha$ in LPS-activated HepG-2 cells. This result is in agreement with previous reports that supernatant of L. plantarum and L. fermentum inhibited TNF- $\alpha$ production in LPS-induced THP-1 monocytic cells (Aoudia et al., 2016). Moreover, extracellular metabolites of L. plantarum prepared from supernatant suppressed TNF- $\alpha$ secretion via attenuation of NF-kB activation in LPS-activated RAW 264.7 macrophage cell line (Chon et al., 2010). TNF- $\alpha$ secretion in HepG-2 cells was dramatically inhibited by viable cells, heat-killed cells, and sonicated cells of $L$. paracasei MSMC39-1. This may be due to the activity of probiotic cells but not the substances produced by the cells. Our results were similar to previous studies reporting that heat-killed cells and sonicated cells of probiotic Lactobacillus reduced TNF- $\alpha$, and IL- 6 secretion in LPS stimulated HepG- 2 cells (Chiu et al., 2013). A recent study revealed that peptidoglycan extracts of $L$. plantarum isolated from human feces modulated TNF- $\alpha$ and IL- 6 in LPS-induced murine RAW264.7 cell (Choi et al., 2019).

\section{Effect of alcohol and $L$. paracasei MSMC39-1 on body weight and liver weight}

To examine the hepatoprotective role of $L$. paracasei MSMC39-1, we used an ethanol-induced hepatitis rat model. Ethanol was administrated by oral gavage into the rats for 8 weeks. Although alcohol administration for 8 weeks decreased body weight, L. paracasei MSMC39-1 treatment significantly enhanced body weight in these rats (Table 1). There was no statistically significant difference in initial body weight, liver weight, and liver-to-body weight ratio of rats in probiotic, alcohol-induced hepatic steatosis, and probiotic treatment groups when compared with the normal control (Table 1). In the hepatitis rat model of this study, body weight and liver weight were examined. After administration of alcohol for 8 weeks, body weight was decreased, liver weight was increased, and liver-to-body weight ratio (\%) exhibited an upward trend; in accordance with Huang et al. (2019), probiotics increased body weight, reduced AST, ALT, IL6 , and TNF- $\alpha$, and modulated intestinal microbiota in alcohol liver disease model (Huang et al., 2019). Moreover, administration of probiotics increased body weight and decreased ALT, AST, IL-1 $\beta$, and TNF- $\alpha$ compared to the alcohol group of a normal diet and high-fat diet in mice (Hong et al., 2015). 


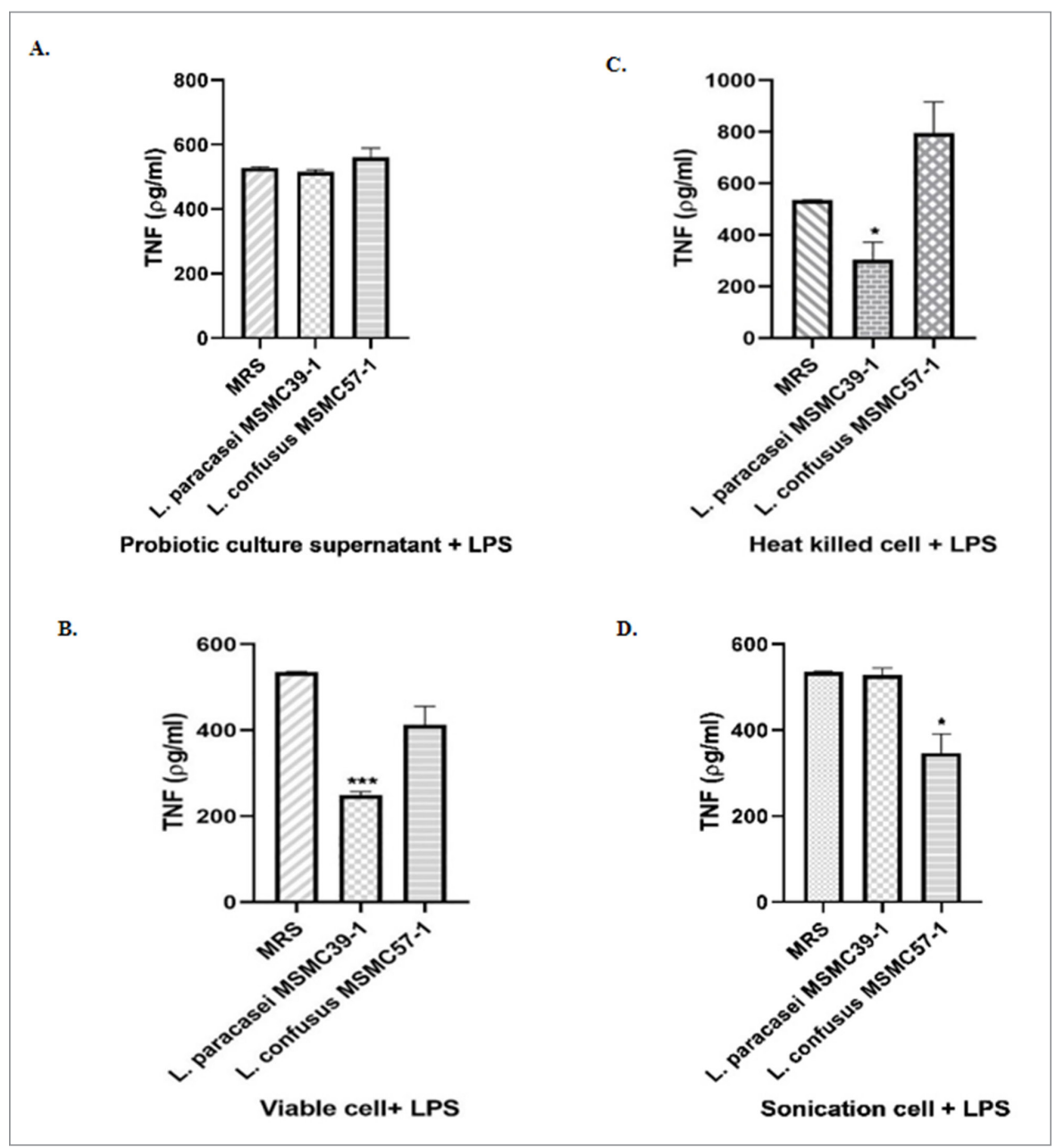

Figure 1. Suppression of TNF- $\alpha$ secretion of (A) probiotic culture supernatant, (B) viable cell, (C) heat-killed cell, and (D) sonication cell of $L$. paracasei MSMC391 and L. confusus MSMC57-1 in HepG-2 cells. Experiments were performed three times, MRS control. Statistical analysis used Student's $t$-test with one-tailed distribution. Error bars indicated standard deviations. ${ }^{*} \rho<0.05 ; * *<0.01 ; * * *<0.001$.

Table 1. Effect of alcohol and L. paracasei MSMC39-1 on body weight and liver weight.

\begin{tabular}{lcccc}
\hline \multirow{2}{*}{ Parameters } & \multicolumn{3}{c}{ Group } \\
\cline { 2 - 5 } & Normal & Probiotic & Alcohol & Probiotic treatment \\
\hline Initial body weight $(\mathrm{g})$ & $237.5 \pm 4.03$ & $239.8 \pm 2.40$ & $239.5 \pm 7.85$ & $239.4 \pm 10.71$ \\
Body weight $(\mathrm{g})$ & $486.4 \pm 6.93$ & $462.5 \pm 25.14$ & $403.1 \pm 10.39^{*}$ & $479.3 \pm 16.5^{\text {** }}$ \\
Liver weight (g) & $15.03 \pm 1.41$ & $14.27 \pm 0.43$ & $15.83 \pm 4.86$ & $15.1 \pm 1.28$ \\
Liver-to-body weight ratio (\%) & $3.08 \pm 0.25$ & $3.09 \pm 0.08$ & $3.94 \pm 1.3$ & $3.15 \pm 0.05$ \\
\hline
\end{tabular}

The data are shown as mean $\pm \mathrm{SD}$. Statistical analysis used one-way ANOVA.

$* \rho<0.05$ compared with the normal groups; ${ }^{* *} \rho<0.05$ compared with the alcohol group.

\section{Effect of $L$. paracasei MSMC39-1 treatment on liver enzyme activity in rats}

In this study, hepatitis rats were treated with viable cells of $L$. paracasei MSMC39-1 for 8 weeks. The level of AST in the serum of the alcohol-treated group was elevated (Fig. 2A). AST level significantly reduced in probiotic-treated hepatitis rat $\left({ }^{*} p<\right.$ 0.05 ) (Fig. 2A) when compared to the alcohol group. The level of ALT in serum of alcohol-induced hepatitis rats was elevated but reduced when $L$. paracasei MSMC39-1 was given to these rats (Fig. 2B). ALT levels in the normal control and L. paracasei
MSMC39-1 groups were lower than that off alcohol group (Fig. 2B). These results suggest the protective role of $L$. paracasei MSMC39-1 on liver disease. AST and ALT are biomarker enzymes of liver function. The ratio of AST and ALT level which is greater than or equal to two is considered as alcoholic hepatitis (Torruellas et al., 2014). In this study, AST levels of alcohol hepatitis rats were two times more than ALT levels in serum. In addition, the probiotic-treated group with viable cells of $L$. paracasei MSMC39-1 showed reductions of AST and ALT levels in serum of hepatitis rats. Similar to other studies, L. plantarum C88, L. 


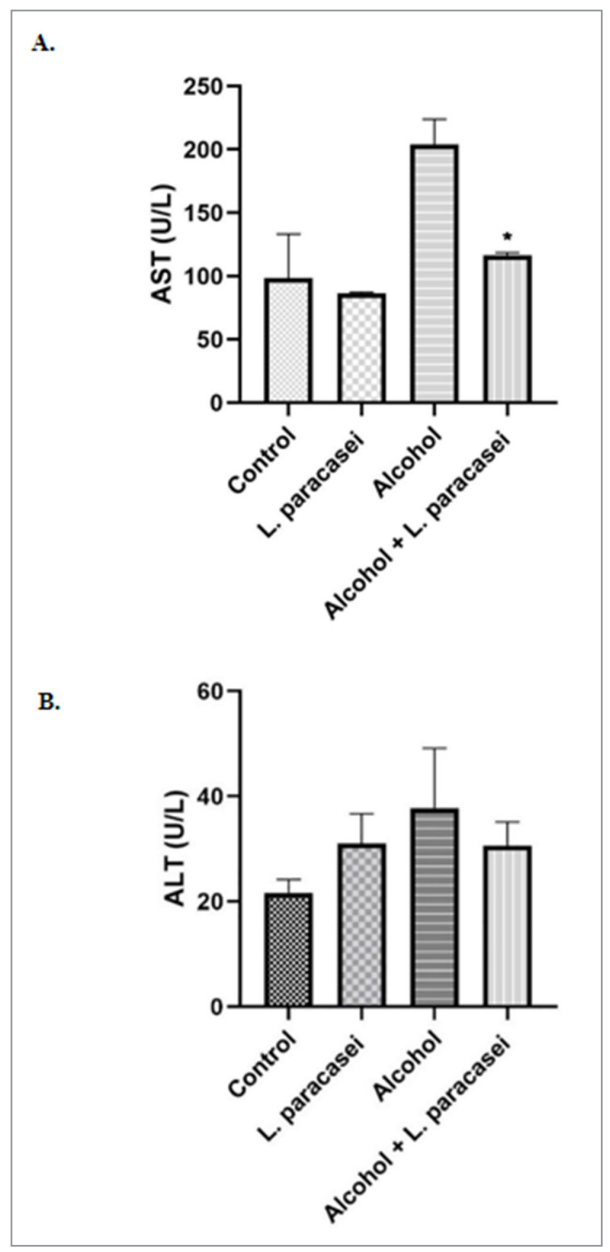

Figure 2. Effect of L. paracasei MSMC39-1 on liver enzyme functional test. (A) AST level; (B) ALT. Error bar indicated standard deviation. Statistical analysis used one-way ANOVA and $* \rho<0.05$; significant differences from the control $(n=6$ rats $)$.

plantarum, L. fermentum, L. helveticus, and L. casei reduced ALT and AST enzymes in the serum (Chen et al., 2018; Wang et al., 2019; Zhao et al., 2014, 2017). Likewise, in the clinical trial study, the levels of AST and ALT were reduced in alcoholic liver disease patients who received probiotics B. bifidum and L. plantarum 8PA3 supplementation (Kirpich et al., 2008). L. paracasei MSMC39-1 improved AST and ALT function which may be due to suppression of proinflammatory cytokine production such as TNF- $\alpha$ in the liver resulting in less inflammation. Similar mechanism, L. plantarum, L. fermentum, and L. plantarum C88 improved liver function enzyme caused by decreasing of TNF- $\alpha$ expression (Chen et al., 2018) and oxidative stress (Zhao et al., 2017).

\section{Effect of $L$. paracasei MSMC39-1 treatment on TNF- $\alpha$ secretion in the liver tissues}

A high level of TNF- $\alpha$ secretion was observed in hepatic tissues of hepatitis rats administered with $30 \%$ alcohol for 8 weeks (Fig. 3). TNF- $\alpha$ level in hepatic tissue of rat in probiotic L. paracasei MSMC39-1-treated group was lower than that of the alcohol control group $(* p<0.05)$ (Fig. 3). A similar level of TNF- $\alpha$ was observed in liver tissues of the normal control and probiotic

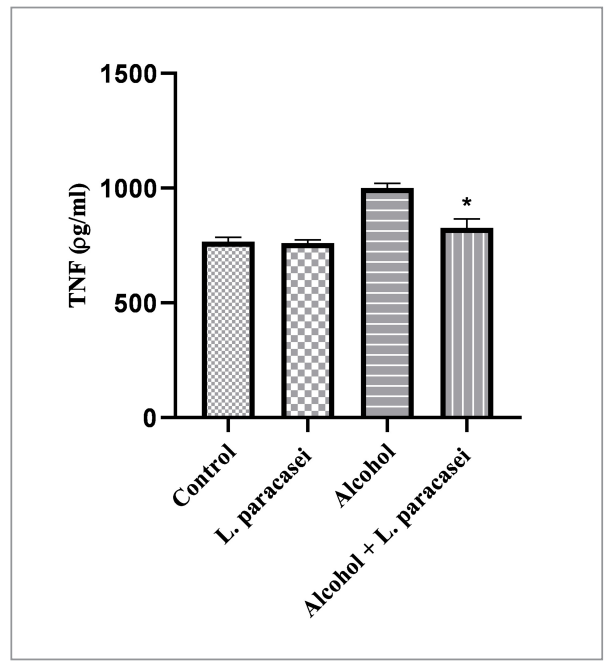

Figure 3. Effect of L. paracasei MSMC39-1 suppresses TNF- $\alpha$ secretion in liver tissue of hepatitis rat. Error bar indicated standard deviation. Statistical analysis used one-way ANOVA and $* \rho<0.05$; significant differences from the control $(n=6$ rats $)$.

control group (Fig. 3). In this study, L. paracasei MSMC391 significantly suppressed TNF- $\alpha$ secretion $(* p<0.05)$ in liver tissue of alcohol hepatitis rats. Our result is similar to previous studies which reported that L. plantarum, L. fermentum, and $L$. helveticus decreased TNF- $\alpha$ and IL-1 $\beta$ gene expression in hepatic tissues of acute liver injury models (Chen et al., 2018; Wang et al., 2019). Moreover, Bifidobacterium longum LC67 and L. plantarum LC27 reduced TNF- $\alpha$ levels in serum and liver of ethanolinduced acute liver damage in mice (Kwon et al., 2017). Wang et al. (2013) revealed that $L$. casei Zhang pretreatment reduced TNF- $\alpha$ expression in LPS/D-galactosamine induced liver injury rat. Moreover, in liver fibrosis rats, Lactobacillus sp. and $\alpha$-lipoic acid reduced TNF- $\alpha$ expression in liver tissue (Zoheir et al., 2014). Ethanol consumption destroys gut barrier function leading to elevation of LPS in the GI tract. This LPS then binds to TLR-4 on Kupffer cells resulting in proinflammatory cytokine production and liver inflammation (Meroni et al., 2019). A study by Xu et al. (2019) reported the role of probiotics on proinflammatory cytokine production. Exopolysaccharides produced by L. buchneri TCP016 reduced proinflammatory cytokine production such as TNF- $\alpha$, IL$1 \beta$, and IL-6 leading to liver protection in LPS/D-galactosamineinduced liver damage in mice. In addition, supernatant of $L$. reuter $i$ reduced liver inflammation by inhibition of IL- 6 and TNF- $\alpha$ production in acute liver injury mice (Cui et al., 2019).

Effect of $L$. paracasei MSMC39-1 treatment on the liver and colon histopathology in alcohol-induced hepatitis rats

Probiotic L. paracasei MSMC39-1 at $1 \times 10^{9} \mathrm{CFU} / \mathrm{ml}$ was administered in alcohol-induced hepatitis rats for 8 weeks and histopathology of liver tissues was examined by hematoxylin and eosin staining. Normal hepatic architectures of liver tissues were observed in normal and probiotic control groups as shown in Figure 4. High levels of fat accumulation in cytoplasm of hepatocytes, swollen hepatocytes, hydropic degeneration, and infiltration of inflammatory cells were prominent in liver tissues of the alcoholic 
hepatitis group. Interestingly, treatment of probiotic L. paracasei MSMC39-1 improved liver tissue condition of hepatitis rats as indicated by normal hepatic architecture and slight fat accumulation in the hepatocytes as shown in Figure 4. Alcoholic hepatitis rats were treated with probiotic L. paracasei MSMC39-1 for 8 weeks and histopathology of colon tissues was examined by hematoxylin and eosin staining. Normal intestinal architectures of colon tissues were observed in normal and probiotic control groups as shown in Figure 5. Crypt edema and infiltration of inflammatory cells were found in alcoholic hepatitis colon tissues of rats administered with $30 \%$ alcohol for 8 weeks. Interestingly, probiotic $L$. paracasei MSMC39-1 treatment improved colon tissues of hepatitis rats with slight crypt edema and fewer inflammatory cells as shown in Figure 5. Chronic alcohol consumption induced lipid accumulation via the AMP-activated protein kinase (AMPK) signaling pathway, resulting in hepatic steatosis ( $\mathrm{Gu}$ et al., 2019). Hepatic steatosis and inflammatory cell infiltration can be observed in liver histology of alcohol hepatitis rats. On the other hand, feeding viable cells of L. paracasei MSMC39-1 reduced hepatic steatosis and the number of inflammatory cells. The reduction of hepatic steatosis by probiotics may be due to the reduction of sterol regulatory element-binding protein 1 (SREBP-1), which plays a key role in lipogenesis and fatty acid oxidation (Gu et al., 2019). Previous animal models showed that oral gavage of L. rhamnosus GG improved leaky gut and reduced colonic and liver inflammation in alcohol-induced liver injury in rats (Forsyth et al., 2009). Similarly, Wang et al. (2012) indicated that cell-free supernatant of L. rhamnosus GG improved liver damage and intestinal inflammation via tight junction protection. In contrast, administration of probiotics $L$. casei, L. plantarum, L. Acidophilus, Lactobacillus delbrueckii subsp. bulgaricus, B. longum, Bifidobacterium breve, Bifidobacterium Infantis, and Streptococcus salivarius subsp. thermophilus (VSL\#3) for 6 months reduced hepatic encephalopathy in liver cirrhosis Indian patients (Dhiman et al., 2014).

\section{Fecal gut microbiota alteration in L. paracasei MSMC39-1 treatment}

Stool was collected after the end of experiments for the detection of gut microbiota alteration. The normal control and probiotic control showed a similar number of genera Lactobacillus and Bifidobacterium as shown in Figure 6. The numbers of E. coli and Proteus were increased in alcohol hepatitis rats, whereas the number of Lactobacillus was decreased in this group as shown in Figure 6. Although administration of L. paracasei MSMC39-1 increased the number of genus Lactobacillus and Bifidobacterium alcohol-induced hepatitis rats, a decrease in the number of $E$. coli and Proteus was observed in these rats when compared with the
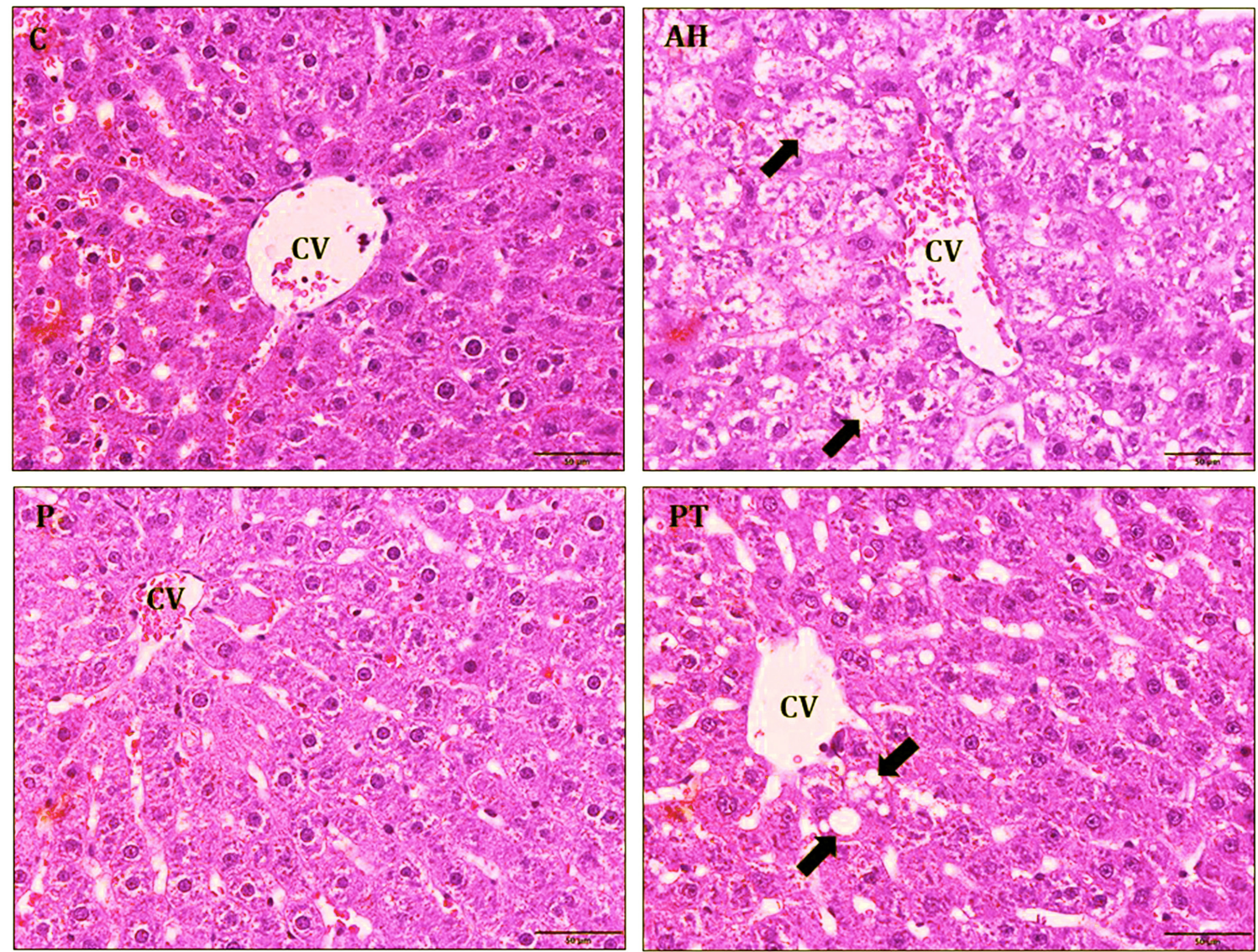

Figure 4. Histology of liver tissue was estimated by hematoxylin and eosin staining in hepatitis rats ( $n=6$ rats). Normal control (C) and probiotic control (P) showed normal hepatocyte and regularity hepatocyte arrangement. Alcoholic hepatitis (AH) indicated that fat accumulation was in the hepatocyte (black arrow) and improved by probiotic $L$. paracasei MSMC39-1 treatment (PT). 400× magnification and scale bar $50 \mu \mathrm{m}$. The tip of portal lobules was the central vein (CV). 

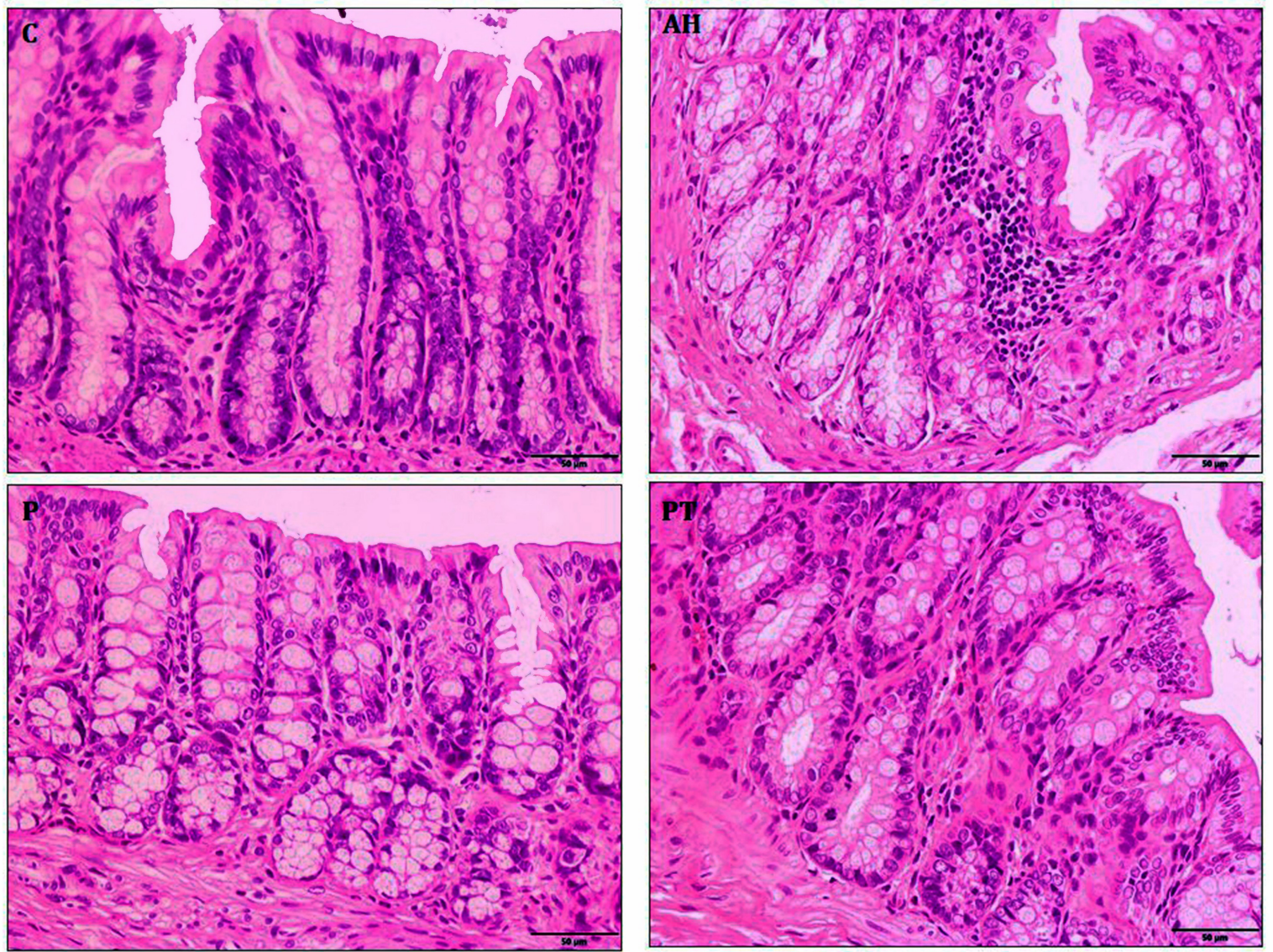

Figure 5. Histology of colon tissue was an estimate by hematoxylin and eosin staining in hepatitis rats. Normal control (C) and probiotic control (P) showed normal crypt. Alcoholic hepatitis (AH) indicated intense inflammatory cell and crypt edema and was improved by probiotic L. paracasei MSMC39-1 treatment (PT). 400× magnification.

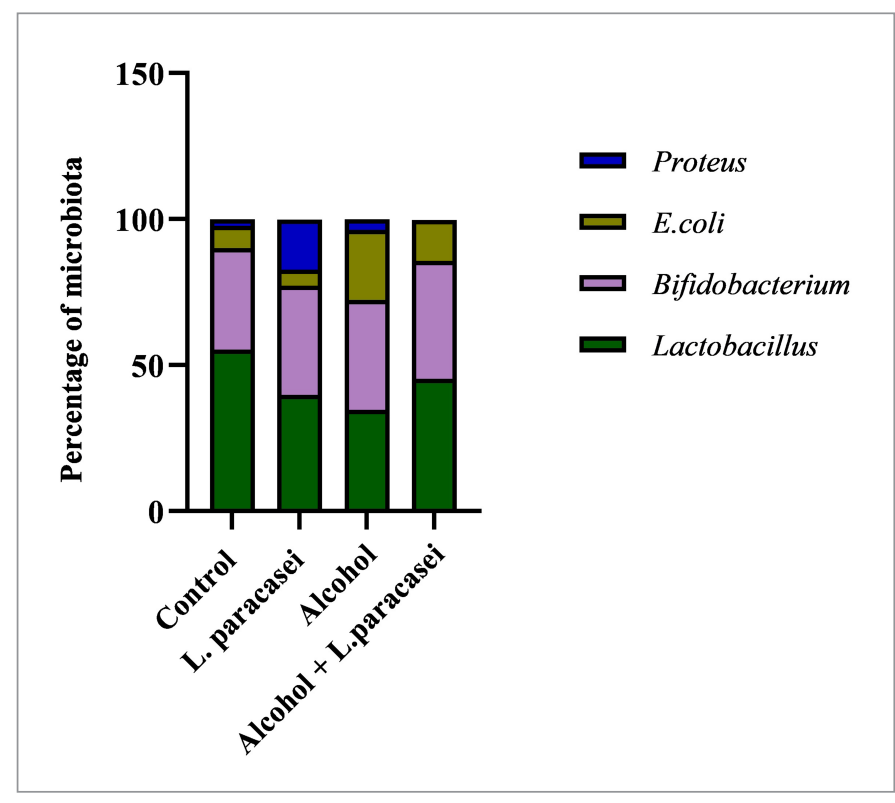

Figure 6. Effect of L. paracasei MSMC39-1 on gut microbiota alteration in hepatitis rats. Statistical analysis used one-way ANOVA and $* \rho<0.05$; significant differences from the control ( $n=6$ rats). alcohol control group (Fig. 6). The data from next-generation sequencing of stool revealed an increase in Bacteroidetes and a decrease in Firmicutes in alcohol hepatitis rats as shown in Figure 7A. After L. paracasei MSMC39-1 treatment in alcohol-induced hepatitis rats, the number of phylum Firmicutes (Lactobacillus spp.) increased whereas the number of Bacteroidetes (Prevotella spp.) decreased when compared to alcohol control as shown in Figure 7A and B. Furthermore, in this study, L. paracasei MSMC39-1 was a potent gut microbiota modulation which increased phylum Firmicutes and decreased Bacteroidetes in alcohol-induced hepatitis rats. In the alcohol alone group, the number of phylum Firmicutes decreased whereas Bacteroidetes increased resulting in dysbiosis in the GI tract (Tian et al., 2020). Modulation of microbiota by probiotics has been reported. Administration of L. buchneri TCP016 modulated gut microbiota in LPS/Dgalactosamine-induced liver damage in mice (Xu et al., 2019). A recent study by Tian et al. (2020) showed that administration of live cells of $B$. breve for 6 weeks was able to modulate gut microbiota in alcohol liver disease mice model via protection of intestinal tight junction and liver inflammation. Likewise, supplementation of LGG modulated intestinal microbiota and alleviated liver injury in mice (Gu et al., 2020). 


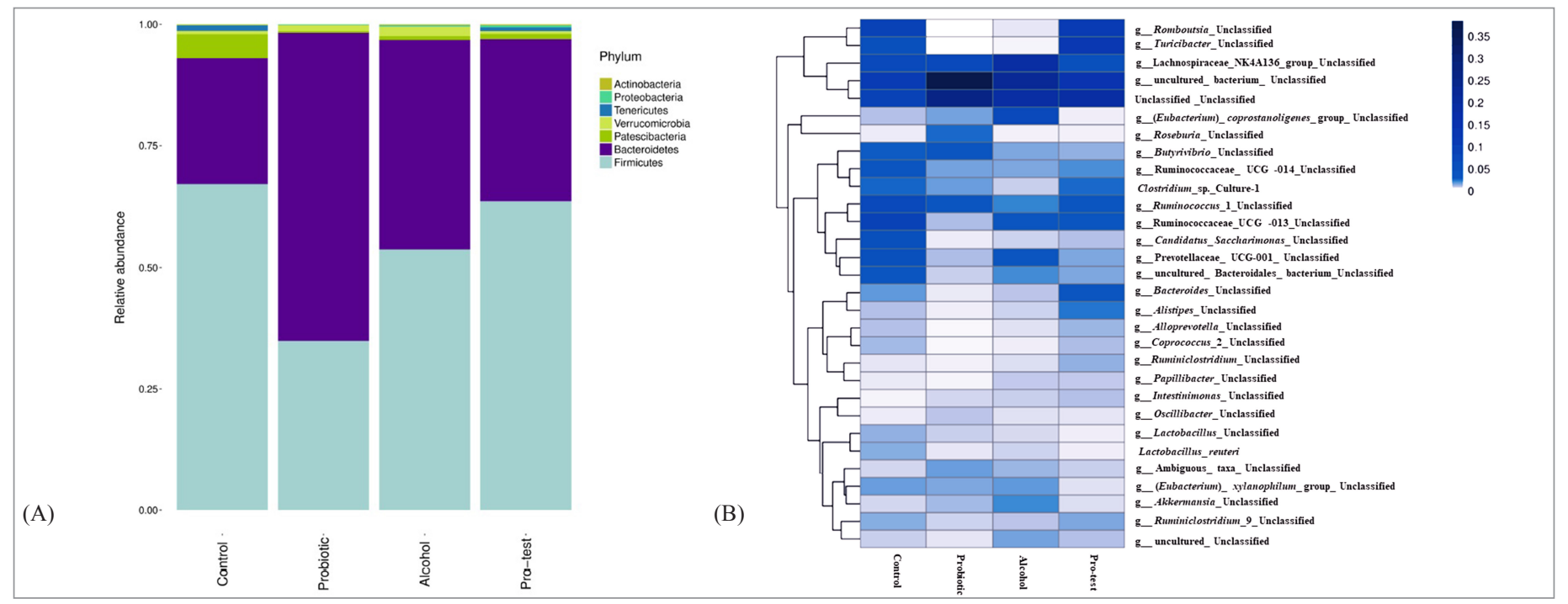

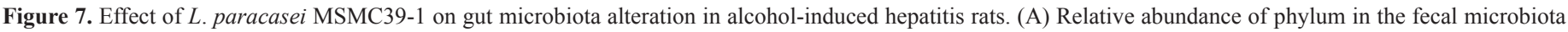

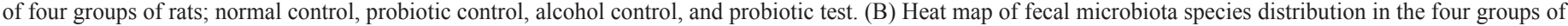

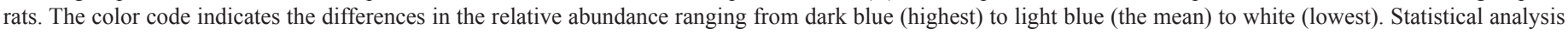
used one-way ANOVA and $* \rho<0.05$; significant differences from the control ( $n=6$ rats).

\section{CONCLUSION}

In conclusion, this finding suggested that probiotic $L$. paracasei MSMC39-1 inhibited TNF- $\alpha$ production in HepG- 2 cell lines and improved liver and colon inflammation. Administration of L. paracasei MSMC39-1 ameliorated the number of beneficial microbes including Lactobacillus and Bifidobacterium in alcoholinduced hepatitis rats. Further studies would confirm the potential roles of L. paracasei MSMC39-1 to protect other liver functions in alcohol liver diseases and inflammation condition diseases.

\section{CONFLICT OF INTEREST}

The authors have no conflicts of interest to disclose.

\section{FUNDING}

This research was funded by research grants (Grants no. 274/2560, no. 384/2561, and no. 152/2563) from the Faculty of Medicine and HRH Princess Maha Chakri Sirindhorn Medical Center, Srinakharinwirot University. Special thanks are due to the Department of Microbiology for providing facilities and scientific equipment.

\section{AUTHOR CONTRIBUTIONS}

All authors made substantial contributions to conception and design, acquisition of data, or analysis and interpretation of data; took part in drafting the article or revising it critically for important intellectual content; agreed to submit to the current journal; gave final approval of the version to be published; and agree to be accountable for all aspects of the work. All the authors are eligible to be an author as per the international committee of medical journal editors (ICMJE) requirements/guidelines.

\section{ETHICAL APPROVALS}

The animal experiment protocol was approved (license no. 9/2561) by the Animal Ethics Committee of the Faculty of Medicine, Srinakharinwirot University.

\section{PUBLISHER'S NOTE}

This journal remains neutral with regard to jurisdictional claims in published institutional affiliation.

\section{REFERENCES}

Aoudia N, Rieu A, Briandet R, Deschamps J, Chluba J, Jego G, Guzzo J. Biofilms of Lactobacillus plantarum and Lactobacillus fermentum: effect on stress responses, antagonistic effects on pathogen growth and immunomodulatory properties. Food Microbiol, 2016; 53:51-9.

Bermudez-Brito M, Plaza-Díaz J, Muñoz-Quezada S, GómezLlorente C, Gil A. Probiotic mechanisms of action. Ann Nutr Metab, 2012; 61(2):160-74.

Bull-Otterson L, Feng W, Kirpich I, Wang Y, Qin X, Liu Y, Gobejishvili L, Joshi-Barve S, Ayvaz T, Petrosino J, Kong M, Barker D, McClain C, Barve S. Metagenomic analyses of alcohol induced pathogenic alterations in the intestinal microbiome and the effect of Lactobacillus rhamnosus GG treatment. PloS One, 2013; 8(1):e53028.

Cai Z, Xu P, Wu Z, Pan D. Anti-inflammatory activity of surface layer protein SlpA of Lactobacillus acidophilus CICC 6074 in LPS-induced RAW 264.7 cells and DSS-induced mice colitis. J Funct Foods, 2018; $51: 16-27$.

Chen X, Zhang, J, Yi R, Mu J, Zhao X, Yang Z. Hepatoprotective effects of Lactobacillus on carbon tetrachloride-induced acute liver injury in mice. Int J Mol Sci, 2018; 19(8):2212.

Cheung TC, Ware CF. Tumor necrosis factor receptors. Encyclopedia of biological chemistry, St. Louis, MO, 2013.

Chiu YH, Lin SL, Ou CC, Lu YC, Huang HY, Lin MY. Antiinflammatory effect of lactobacilli bacteria on HepG2 cells is through crossregulation of TLR4 and NOD2 signalling. J Funct Foods, 2013; 5(2):82028 .

Choi EA, Chang HC. Cholesterol-lowering effects of a putative probiotic strain Lactobacillus plantarum EM isolated from kimchi. LWT Food Sci Technol, 2015; 62(1):210-17.

Choi SH, Lee SH, Kim MG, Lee HJ, Kim GB. Lactobacillus plantarum CAU1055 ameliorates inflammation in lipopolysaccharideinduced RAW264.7 cells and a dextran sulfate sodium-induced colitis animal model. J Dairy Sci, 2019; 102(8):6718-25.

Chon H, Choi B, Jeong G, Lee E, Lee S. Suppression of proinflammatory cytokine production by specific metabolites of 
Lactobacillus plantarum 10hk2 via inhibiting NF-kB and p38 MAPK expressions. Comp Immunol Microbiol Infect Dis, 2010; 33(6):e41-9.

Chu WM. Tumor necrosis factor. Cancer Lett, 2013; 328(2):

$222-25$.

Cui Y, Qi S, Zhang W, Mao J, Tang R, Wang C, Liu J, Luo XM, Wang H. Lactobacillus reuteri ZJ617 culture supernatant attenuates acute liver injury induced in mice by lipopolysaccharide. J Nutr 2019; 149(11):2046-55.

Das D, Goyal A. Antioxidant activity and $\gamma$-aminobutyric acid (GABA) producing ability of probiotic Lactobacillus plantarum DM5 isolated from Marcha of Sikkim. LWT Food Sci Technol, 2015; 61(1):263-68.

Dhiman RK, Rana B, Agrawal S, Garg A, Chopra M, Thumburu KK, Chawla YK. Probiotic VSL\#3 reduces liver disease severity and hospitalization in patients with cirrhosis: a randomized, controlled trial. Gastroenterology, 2014; 147(6):1327-37.

Fang TJ, Guo JT, Lin MK, Lee MS, Chen YL, Lin WH. Protective effects of Lactobacillus plantarum against chronic alcoholinduced liver injury in the murine model. Appl Microbiol Biotechnol, 2019; 103(20):8597-608.

Forsyth CB, Farhadi A, Jakate SM, Tang Y, Shaikh M, Keshavarzian A. Lactobacillus GG treatment ameliorates alcohol-induced intestinal oxidative stress, gut leakiness, and liver injury in a rat model of alcoholic steatohepatitis. Alcohol, 2009; 43(2):163-72.

Fung P, Pyrsopoulos N. Emerging concepts in alcoholic hepatitis. World J Hepatol, 2017; 9(12):567-85.

Gao K, Wang C, Liu L, Dou X, Liu J, Yuan L, Zhang W, Wang H. Immunomodulation and signaling mechanism of Lactobacillus rhamnosus GG and its components on porcine intestinal epithelial cells stimulated by lipopolysaccharide. J Microbiol Immunol Infect, 2017; 50(5):700-13.

Gu Z, Liu Y, Hu S, You Y, Wen J, Li W, Wang Y. Probiotics for alleviating alcoholic liver injury. Gastroenterol Res Pract, 2019; 2019:9097276.

Gu Z, Wu Y, Wang Y, Sun H, You Y, Piao C, Wang Y. Lactobacillus rhamnosus granules dose-dependently balance intestinal microbiome disorders and ameliorate chronic alcohol-induced liver injury. J Med Food, 2020; 23(2):114-24.

Hong M, Kim SW, Han SH, Kim DJ, Suk KT, Kim YS, Ham YL. Probiotics (Lactobacillus rhamnosus R0011 and acidophilus R0052) reduce the expression of toll-like receptor 4 in mice with alcoholic liver disease. PLoS One, 2015; 10(2):1-17.

Huang CH, Ho CY, Chen CT, Hsu HF, Lin YH. Probiotic BSH activity and anti-obesity potential of Lactobacillus plantarum strain TCI378 isolated from Korean Kimchi. Prev Nutr Food Sci, 2019; 24(4):434-41.

Huang $\mathrm{H}$, Lin Z, Zeng Y, Lin X, Zhang Y. Probiotic and glutamine treatments attenuate alcoholic liver disease in a rat model. Exp Ther Med, 2019; 18(6):4733-39.

Kim IS, Lee SH, Kwon YM, Adhikari B, Kim JA, Yu DY, Kim GI, Lim JM, Kim SH, Lee SS, Moon YS, Choi IS, Cho KK. Oral administration of beta-glucan and Lactobacillus plantarum alleviates atopic dermatitis-like symptoms. J Microbiol Biotechnol, 2019; 29(11):1693-706.

Kim WK, Jang YJ, Seo B, Han DH, Park SJ, Ko GP. Administration of Lactobacillus paracasei strains improves immunomodulation and changes the composition of gut microbiota leading to improvement of colitis in mice. J Funct Foods, 2019; 52:565-75.

Kirpich IA, Solovieva NV, Leikhter SN, Shidakova NA, Lebedeva OV, Sidorov PI, Cave M. Probiotics restore bowel flora and improve liver enzymes in human alcohol-induced liver injury: a pilot study. Alcohol, 2008; 42(8):675-82.

Kwon EK, Kang GD, Kim WK, Han MJ, Kim DH. Lactobacillus plantarum LC27 and Bifidobacterium longum LC67 simultaneously alleviate ethanol-induced gastritis and hepatic injury in mice. J Funct Foods, 2017; 38:389-98.

Ladda B, Theparee T, Chimchang J, Tanasupawat S, Taweechotipatr M. In vitro modulation of tumor necrosis factor $\alpha$ production in THP-1 cells by lactic acid bacteria isolated from healthy human infants. Anaerobe, 2015; 33:109-16.
Leggio L, Lee MR. Treatment of alcohol use disorder in patients with alcoholic liver disease. Am J Med, 2017; 130(2):124-34.

Meroni M, Longo M, Dongiovanni P. Alcohol or gut microbiota: who is the guilty? Int J Mol Sci, 2019; 20(18):1-22.

Miao L, Jiang $\mathrm{T}$, Lin $\mathrm{S}$, Jin $\mathrm{T}$, Hu J, Zhang M, Liu G. aggregation, adherence, anti-adhesion and antagonistic activity properties relating to surface charge of probiotic Lactobacillus brevis gp104 against Staphylococcus aureus. Microb Pathog, 2020; 147:104420.

Niederreiter L, Tilg H. Cytokines and fatty liver diseases. Liver Res, 2018; 2(1):14-20.

Oh NS, Joung JY, Lee JY, Kim Y. Probiotic and anti-inflammatory potential of Lactobacillus rhamnosus 4B15 and Lactobacillus gasseri 4M13 isolated from infant feces. PLoS One, 2018; 13(2):e0192021.

Pan Y, Wang H, Tan F, Yi R, Li W, Long X, Zhao X. Lactobacillus plantarum KFY02 enhances the prevention of CCl4-induced liver injury by transforming geniposide into genipin to increase the antioxidant capacity of mice. J Funct Foods, 2020; 73:104128.

Pérez-Burillo S, Pastoriza S, Gironés A, Avellaneda A, Pilar Francino M, Rufián-Henares JA. Potential probiotic salami with dietary fiber modulates metabolism and gut microbiota in a human intervention study. J Funct Foods, 2020; 66:103790.

Rabah Ha. Beneficial propionibacteria within a probiotic emmental cheese: impact on dextran sodium sulphate-induced colitis in mice. Microorganisms, 2020; 8(3):380.

Shewale RN, Sawale PD, Khedkar CD, Singh A. Selection criteria for probiotics: a review. Int J Probiotics Prebiotics, 2014; 9(12):17-22.

Tian X, Li R, Jiang Y, Zhao F, Yu Z, Wang Y, Li X. Bifidobacterium breve ATCC 15700 pretreatment prevents alcoholic liver disease through modulating gut microbiota in mice exposed to chronic alcohol intake. $\mathrm{J}$ Funct Foods, 2020; 72:104045.

Torruellas C, French SW, Medici V. Diagnosis of alcoholic liver disease. World J Gastroenterol, 2014; 20(33):11684-99.

Wang B, Yao M, Lv L, Ling Z, Li L. The human microbiota in health and disease. Engineering, 2017; 3(1):71-82.

Wang Q, Lv L, Jiang H, Wang K, Yan R, Li Y, Li L. Lactobacillus helveticus R0052 alleviates liver injury by modulating gut microbiome and metabolome in d-galactosamine-treated rats. Appl Microbiol Biotechnol, 2019; 103:9673-86.

Wang Y, Li Y, Xie J, Zhang Y, Wang J, Sun X, Zhang H Protective effects of probiotic Lactobacillus casei Zhang against endotoxinand d-galactosamine-induced liver injury in rats via anti-oxidative and antiinflammatory capacities. Int Immunopharmacol, 2013. 15(1):30-7.

Wang Y, Liu Y, Kirpich I, Ma Z, Wang C, Zhang M, Feng W. Lactobacillus rhamnosus GG reduces hepatic TNF $\alpha$ production and inflammation in chronic alcohol-induced liver injury. J Nutr Biochem, 2013; 24(9):1609-15.

Wang Y, Liu Y, Sidhu A, Ma Z, McClain C, Feng W. Lactobacillus rhamnosus GG culture supernatant ameliorates acute alcohol-induced intestinal permeability and liver injury. Am J Physiol Gastrointest Liver Physiol, 2012; 303(1):G32-41.

Wang Y, Xie J, Li Y, Dong S, Liu H, Chen J, Zhang H. Probiotic Lactobacillus casei Zhang reduces pro-inflammatory cytokine production and hepatic inflammation in a rat model of acute liver failure. Eur J Nutr, 2016; 55(2):821-31.

WHO/FAO. Probiotics in food: Health and nutritional properties and guidelines for evaluation. FAO Food Nutr, pp 1-11, 2002.

$\mathrm{Xu}$ R, Aruhan, Xiu L, Sheng S, Liang Y, Zhang H, Wang X. Exopolysaccharides from Lactobacillus buchneri TCP016 attenuate LPSand D -galn-induced liver injury by modulating the gut microbiota. J Agric Food Chem, 2019; 67(42):11627-37.

Zhang X, Wu Y, Wang Y, Wang X, Piao C, Liu J, Liu Y, Wang $\mathrm{Y}$, The protective effects of probiotic-fermented soymilk on high-fat dietinduced hyperlipidemia and liver injury. J Funct Foods, 2017; 30:220-27.

Zhang Z, Zhou H, Bai L, Lv Y, Yi H, Zhang L, Li R. Protective effects of probiotics on acute alcohol-induced liver injury in mice through 
alcohol metabolizing enzymes activation and hepatic TNF- $\alpha$ response reduction. J Funct Foods, 2019; 59:234-41.

Zhao L, Jiang Y, Ni Y, Zhang T, Duan C, Huang C, Li S. Protective effects of Lactobacillus plantarum C88 on chronic ethanolinduced liver injury in mice. J Funct Foods, 2017; 35:97-104.

Zhao ZW, Pan DD, Wu Z, Sun YY, Guo YX, Zeng XQ. Antialcoholic liver activity of whey fermented by Lactobacillus casei isolated from koumiss. J Dairy Sci, 2014; 97(7):4062-71.

Zoheir KMA, Amara AA, Ahmad SF, Mohammad MA, Ashour AE, Harisa GI, Abd-Allah AR. Study of the therapeutic effects of Lactobacillus and $\alpha$-lipoic acid against dimethylnitrosamine-induced liver fibrosis in rats. J Genet Eng Biotechnol, 2014; 12(2):135-42.
How to cite this article:

Ladda B, Tangteerawatana P, Padungchaichot P, Pradidarcheep W, Kasorn A, Taweechotipatr M. Anti-inflammatory effect of probiotic Lactobacillus paracasei MSMC39-1 on alcoholinduced hepatitis in rats. J Appl Pharm Sci, 2021; 11(04): 046-056. 